PROCEEDINGS OF THE

AMERICAN MATHEMATICAL SOCIETY

Volume 133, Number 4, Pages 1175-1184

S 0002-9939(04)07622-1

Article electronically published on October 14, 2004

\title{
LOCAL DUAL AND POLY-SCALE REFINABILITY
}

\author{
QIYU SUN
}

(Communicated by David R. Larson)

\begin{abstract}
For a compactly supported function $f$, let $S_{n}(f), n \geq 0$, be the space of all finite linear combinations of $f\left(M^{n} \cdot-k\right), k \in \mathbf{Z}$. In this paper, we consider the explicit construction of local duals of $f$ and the poly-scale refinability of functions in $S_{0}(f)$ when $f$ is an $M$-refinable function. We show that for any $M$-refinable function $f$, there exists a local dual of $f$ in $S_{N}(f)$ for some $N \geq 0$, and that any function in $S_{0}(f)$ is poly-scale refinable.
\end{abstract}

\section{INTRODUCTION}

Let $L^{2}:=L^{2}(\mathbf{R})$ be the space of all square integrable functions on $\mathbf{R}$ and $\langle\cdot, \cdot\rangle$ be the usual inner product on $L^{2}$. For compactly supported functions $f$ and $g$ in $L^{2}$, we say that $g$ is a local dual of $f$ if

$$
\left\langle f(\cdot-k), g\left(\cdot-k^{\prime}\right)\right\rangle=\delta_{k k^{\prime}} \quad \forall k, k^{\prime} \in \mathbf{Z},
$$

where $\delta$ is the usual Kronecker symbol. Given a compactly supported function $f$, we define the corresponding semi-convolution $f *^{\prime}$ on $\ell$, the space of all sequences on $\mathbf{Z}$, by

$$
f *^{\prime}: \ell \ni\{c(k)\} \longmapsto \sum_{k \in \mathbf{Z}} c(k) f(\cdot-k) .
$$

We see that for any $k \in \mathbf{Z}$, the $k$ th component of a sequence $c$ contributes only to $f *^{\prime} c$ at the neighborhood $k+\operatorname{supp} f$ of the location $k$, and hence the semiconvolution is locally defined. If there exists a local dual $g$ of $f$, then for any $k \in \mathbf{Z}$, the $k$-th component $c(k)$ of the sequence $c:=\{c(k)\}$ can be recovered from the restriction of the semi-convolution $f *^{\prime} c$ to a neighborhood of the location $k$ in a stable way because

$$
c(k)=\left\langle f *^{\prime} c, g(\cdot-k)\right\rangle \quad \forall k \in \mathbf{Z},
$$

and hence the semi-convolution $f *^{\prime}$ has a local inverse. From (1.2), we see that the semi-convolution $f *^{\prime}$ is one-to-one, which is known as linear independent shifts of $f(10,13)$. Conversely if $f$ has linear independent shifts, then a local dual $g$ of $f$ can be found ([1, 9, 14]). Thus for any given compactly supported $L^{2}$ function $f$, the existence of its local dual in $L^{2}$ is equivalent to its linear independent shifts.

Received by the editors December 17, 2002 and, in revised form, December 8, 2003.

2000 Mathematics Subject Classification. Primary 42C40, 41A65.

Key words and phrases. Local dual, linear independent shifts, refinability, poly-scale refinability.

Partial results of this paper were announced in the 2002 Fall Southeastern Section Meeting of AMS, Orlando, November 9-10, 2002.

(C)2004 American Mathematical Society 
We say that a compactly supported function $f$ is $M$-refinable, or refinable for short, if

$$
f=\sum_{k \in \mathbf{Z}} a(k) f(M \cdot-k) \text { and } \hat{f}(0)=1,
$$

where the dilation $M$ is a fixed integer larger than 2 , and $\{c(k)\}$ is a finitely supported sequence on $\mathbf{Z}$ and satisfies $\sum_{k \in \mathbf{Z}} a(k)=M$ ([2] 5, 12]). Here the Fourier transform $\hat{f}$ of an integrable function $f$ is defined by $\hat{f}(\xi)=\int_{\mathbf{R}} f(x) e^{-i x \xi} d x$. The sequence $\{a(k)\}$ and the function $H(\xi)=\frac{1}{M} \sum_{k \in \mathbf{Z}} a(k) e^{-i k \xi}$ are called the mask and the symbol of the refinable function $f$ respectively. The first topic considered in this paper is the explicit construction of a local dual of a given refinable function. Our motivation is based on the following three easy observations: the first observation is that if $f$ has orthonormal shifts, that is, (1.1) holds for $g$ being replaced by $f$, then $f$ itself is the local dual of $f$, and hence there is a local dual in the shift-invariant space $S_{0}(f)$ generated by $f$,

$$
S_{0}(f)=\left\{f *^{\prime} c: c \in \ell_{0}\right\},
$$

where $\ell_{0}$ is the space of all finitely supported sequences on $\mathbf{Z}$; the second observation is that if $f$ is $M$-refinable, then the spaces

$$
S_{n}(f)=\left\{h\left(M^{n} \cdot\right): h \in S_{0}(f)\right\}, n \geq 0,
$$

satisfy the following nestedness condition:

$$
S_{0}(f) \subset S_{1}(f) \subset \cdots \subset S_{n}(f) \subset \cdots \rightarrow L^{2} ;
$$

and the third observation is that for the hat function $h(x)=\max (1-|x|, 0)$, the function $-\frac{1}{2}(h(2 x-1)+h(2 x+1))+3 h(2 x)$ in $S_{1}(h)$ is a local dual of $h$, where we set $M=2$. The above three observations inspire us to consider the problem whether for any refinable function $f$ we can find its local dual in $S_{N}(f)$ for some $N \geq 0$. An affirmative answer to the above problem for $M=2$ follows from the intertwining multiresolution analysis in 8 . In this paper, we give an affirmative answer to the above problem for any dilation $M$, and prove the following result where the local dual can be chosen from the dilation of the shifts of another refinable function.

Theorem 1.1. Let $f$ and $g$ be compactly supported refinable functions in $L^{2}$. If $f$ and $g$ have linear independent shifts, then there is an $h \in S_{N}(g)$ for some $N \geq 0$ so that $h$ is a local dual of $f$.

From Theorem 1.1, we see that for any refinable function $f \in L^{2}$ with linear independent shifts, a spline of order $L$ with knots on $\mathbf{Z} / M^{N}$ can be a local dual of $f$ when $N$ is sufficiently large. For the case that both $f$ and $g$ are B-splines, a function in $S_{1}(g)$ can be chosen to be a local dual of $f$ from the proof of Theorem 1.1. For instance, $3 \chi_{[0,1 / 2]}-\chi_{[1 / 2,1)}$ is a local dual of the hat function $h$, where we set $M=2$ and we denote the characteristic function on a measurable set $E$ by $\chi_{E}$.

Given any compactly supported refinable function $f \in L^{2}$ with linear independent shifts, the functions in $S_{N}(f)$ have been used in the construction of orthonormal wavelets $([2]$ 5, 12]), tight affine frames $([3,4,6])$, and also the local dual (Theorem 1.1). The second topic of this paper is the refinability of functions in $S_{0}(f)$. Given a refinable function $f$, a function $g \in S_{0}(f)$ is not refinable in general. In particular, one may easily verify that $g=\sum_{k \in \mathbf{Z}} d(k) f(\cdot-k) \in S_{0}(f)$ is refinable if and only if $D\left(z^{M}\right) H(z) / D(z)$ is a Laurent polynomial, where $D(z)=\sum_{k \in \mathbf{Z}} d(k) z^{k}$ 
and $H(z)=\sum_{k \in \mathbf{Z}} a(k) z^{k}$ with $\{a(k)\}$ being the mask of the refinable function $f$. Recently, poly-scale refinability, a weak concept of refinability, was introduced by Dekel and Dyn ([7]). For a compactly supported function $f$, we say that $f$ is poly-scale refinable if

$$
f=\sum_{n=1}^{N} \sum_{k \in \mathbf{Z}} c_{n}(k) f\left(M^{n} \cdot-k\right)
$$

for some $N \geq 1$, where $\left\{c_{n}(k)\right\} \in \ell_{0}, 1 \leq n \leq N$. Clearly, a refinable function is poly-scale refinable, and a compactly supported function $f$ is poly-scale refinable if and only if

$$
S_{0}(f) \subset S_{1}(f)+\cdots+S_{N}(f)
$$

for some $N \geq 1$. In this paper, we show that given a refinable function $f$ having linear independent shifts, any function $g \in S_{0}(f)$ is poly-scale refinable.

Theorem 1.2. Let $f$ be a compactly supported refinable function. Then any function in $S_{0}(f)$ is poly-scale refinable.

Given a compactly supported refinable function $f$ and a function $g \in S_{0}(f)$, we see from Theorem 1.2 that $S_{0}(g) \subset S_{1}(g)+S_{2}(g)+\cdots+S_{N}(g)$ for some $N \geq 1$. Also we notice that $S_{0}(g) \subset S_{0}(f)$. The above two observations inspire us to consider the following problem: whether

$$
S_{0}(f) \subset S_{1}(g)+\cdots+S_{N}(g)
$$

holds for some $N \geq 1$.

Theorem 1.3. Let $f$ be a compactly supported refinable distribution having linear independent shifts, and let $g=\sum_{k \in \mathbf{Z}} d(k) f(\cdot-k) \in S_{0}(f)$ for some nonzero sequence $\{d(k)\} \in \ell_{0}$. Assume that there does not exist $\xi_{0} \in \mathbf{R}$ so that $d\left(M^{n} \xi_{0}\right)=0$ for all $n \geq 0$, where we set $d(\xi):=\sum_{k \in \mathbf{Z}} d(k) e^{-i k \xi}$. Then (1.5) holds for some positive integer $N$.

The condition on $d$ in Theorem 1.3 cannot be dropped out in general. For instance, if $g=f-f(\cdot-1)$, then any function $h$ in $S_{N}(g), N \geq 1$, satisfies $\int_{\mathbf{R}} h(x) d x=0$, which implies that (1.5) does not hold for any $N \geq 1$.

\section{LOCAL DUAL}

In this section, we give a constructive proof to the following slight generalization of Theorem 1.1

Theorem 2.1. Let $f$ be a compactly supported refinable function in $L^{2}$ having linear independent shifts, and let $g$ be a compactly supported function in $L^{2}$, have linear independent shifts and satisfy $\int_{\mathbf{R}} g(x) d x \neq 0$. Then there exists a function $h$ in $S_{N}(g)$ for some $N \geq 0$ so that $h$ is a local dual of $f$.

We remark that the condition $\int_{\mathbf{R}} g(x) d x \neq 0$ in Theorem 2.1 cannot be dropped out in general. For instance, let $f=\chi_{[0,1)}$ and $g=\chi_{[-1 / 2,0)}-\chi_{[0,1 / 2]}$. If we can find $h \in S_{N}(g), N \geq 0$, so that $h$ is a local dual of $f$, then

$$
\langle f(\cdot-k), h\rangle=\delta_{k}, k \in \mathbf{Z} \text {. }
$$

Write $h=\sum_{k \in \mathbf{Z}} a_{N}(k) g\left(2^{N} \cdot-k\right)$ for some $\left\{a_{N}(k)\right\} \in \ell_{0}$. Then direct calculation yields

$$
\langle f(\cdot-k), h\rangle=2^{-N} a_{N}\left(2^{N} k\right)-2^{-N} a_{N}\left(2^{N}(k+1)\right), k \in \mathbf{Z} .
$$


By (2.1) and (2.2), the sequence $\left\{a_{N}(k)\right\}$ is not finitely supported, which is a contradiction.

We say that $\xi_{0}$ is an m-symmetric root of a trigonometric polynomial $a$ if $a\left(\xi_{0}+2 k \pi / m\right)=0$ for all $k \in \mathbf{Z}$. To prove Theorem 2.1, we need a lemma about $m$-symmetric roots of a trigonometric polynomial.

Lemma 2.2. Let $f$ be a compactly supported refinable function having linear independent shifts, and let $A$ be a nonzero trigonometric polynomial. Let $H$ be the symbol of $f$, and define

$$
H_{n}(\xi)=H\left(M^{n-1} \xi\right) \cdots H(\xi)
$$

for $n \geq 1$. Denote the set of all $M^{n}$-symmetric roots of $H_{n} A$ by $K_{n}$ with multiplicity being not counted, where we say that $a$ and $b$ are two different $M^{n}$-symmetric roots if $a-b \notin 2 M^{-n} \pi \mathbf{Z}$. Then $K_{n} \subset 2 M^{-n} \pi \mathbf{Z}$ for sufficiently large $n$.

Proof. Let $f$ satisfy the refinement equation

$$
f=\sum_{k \in \mathbf{Z}} a(k) f(M \cdot-k),
$$

where $\{a(k)\} \in \ell_{0}$ satisfies $\sum_{k \in \mathbf{Z}} a(k)=M$. Taking the Fourier transform of both sides of the equation (2.4), we get

$$
\hat{f}(\xi)=H(\xi / M) \hat{f}(\xi / M)
$$

and using (2.5) iteratively $n$ times, we obtain

$$
\hat{f}(\xi)=H_{n}\left(\xi / M^{n}\right) \hat{f}\left(\xi / M^{n}\right) .
$$

Recall that if $f$ has linear independent shifts, then for any complex number $\xi_{0}$ there exists an integer $k:=k\left(\xi_{0}\right)$ so that $\hat{f}\left(\xi_{0}+2 k \pi\right) \neq 0([10,13])$. This together with (2.6) concludes that

$$
H_{n}(\xi) \text { does not have any } M^{n} \text {-symmetric root for any } n \geq 1 \text {. }
$$

Let $Z(A)$ be the set of all roots of $A(\xi)$ with multiplicity being not counted. By (2.7), we have

$$
K_{n} \subset Z(A)+2 M^{-n} \pi \mathbf{Z}
$$

and hence

$$
\# K_{n}<\infty
$$

for all $n \geq 1$. Here $\# E$ is the cardinality of a finite set $E$.

For any $\xi_{0} \in K_{n}$, let $k_{1}$ be an integer so chosen that $H\left(M^{n-1} \xi_{0}+2 k_{1} \pi / M\right) \neq 0$, and set $\xi_{0}^{\prime}=\xi_{0}+2 M^{-n} k_{1} \pi$. Then, since $H_{n}\left(\xi_{0}+2 M^{-n} k \pi\right) A\left(\xi_{0}+2 M^{-n} k \pi\right)=0$ for all $k \in \mathbf{Z}$ and $H_{n}(\xi)=H\left(M^{n-1} \xi\right) H_{n-1}(\xi)$, we have $\xi_{0}^{\prime} \in K_{n-1}$. Also, one may verify that if $\xi_{0}, \xi_{0}^{\prime} \in K_{n}$ are two different $M^{n}$-symmetric roots of $H_{n} A$, then $\xi_{0}+2 M^{-n} k_{1} \pi$ and $\xi_{0}^{\prime}+2 M^{-n} k_{1}^{\prime} \pi$ are two different $M^{n-1}$-symmetric roots of $H_{n-1} A$, where $k_{1}, k_{1}^{\prime} \in \mathbf{Z}$. Therefore we conclude that

$$
\# K_{n} \leq \# K_{n-1} \quad \forall n \geq 1 \text {. }
$$

Moreover, we see that $\# K_{n}=\# K_{n-1}$ if and only if

$$
K_{n-1}=\left\{\xi_{0}+2 M^{-n} k\left(\xi_{0}\right) \pi: \xi_{0} \in K_{n}\right\},
$$

where $k\left(\xi_{0}\right) \in \mathbf{Z}$ is chosen so that

$$
H\left(M^{n-1} \xi_{0}+2 k \pi / M\right)=0 \quad \forall k \notin k\left(\xi_{0}\right)+M \mathbf{Z},
$$


and

$$
H\left(M^{n-1} \xi_{0}+2 k \pi / M\right) \neq 0 \quad \forall k \in k\left(\xi_{0}\right)+M \mathbf{Z} .
$$

By (2.10) and (2.11), there exist an integer $N_{0}$ and a finite set $K$ such that

$$
K_{n}=K \text { modulo } 2 M^{-n} \pi \mathbf{Z}
$$

for all $n \geq N_{0}$.

For any $\xi_{0} \in K$ and $n \geq N_{0}, H\left(M^{n} \xi_{0}+2 k \pi / M\right)=0$ for $k \in \mathbf{Z} \backslash M \mathbf{Z}$ and $H\left(M^{n} \xi_{0}+2 k \pi / M\right) \neq 0$ for $k \in M \mathbf{Z}$ by (2.12) and (2.13). Then for any $1 \leq k \leq$ $M-1,\left\{M^{n} \xi_{0}+2 k \pi / M: 1 \leq k \leq M-1, n \geq N_{0}\right\}$ is a finite set modulo $2 \pi \mathbf{Z}$, which implies that $M^{n_{1}} \xi_{0}+2 k \pi / M=M^{n_{2}} \xi_{0}+2 k \pi / M \bmod 2 \pi \mathbf{Z}$ for some integers $N_{0} \leq n_{1}<n_{2}$. Therefore $\eta_{0}:=M^{n_{1}} \xi_{0}$ is rational and we may assume that

$$
\eta_{0}=2 j_{0} \pi /(m-1)
$$

for some $0 \leq j_{0} \leq m-2$, where $m=M^{n_{2}-n_{1}}$, and

$$
H\left(M^{n} \eta_{0}+2 k \pi / M\right)=0 \quad \forall k \in \mathbf{Z} \backslash M \mathbf{Z} \quad \text { and } \quad n \geq 0 .
$$

From (2.16), it follows that

$$
H_{n_{2}-n_{1}}\left(\eta_{0}+2 k \pi / m\right)=H\left(M^{n_{2}-n_{1}-1}\left(\eta_{0}+2 k \pi / m\right)\right) \cdots H\left(\eta_{0}+2 k \pi / m\right)=0
$$

for all $k \in \mathbf{Z} \backslash m \mathbf{Z}$, which implies that for any $k \in \mathbf{Z}$ with $k=j_{0}+\cdots+m^{l-1} j_{0}+m^{l} k^{\prime}$ for some $l \geq 0$ and $k^{\prime}-j_{0} \in m \mathbf{Z}$, we have

$$
\begin{aligned}
\hat{f}\left(\eta_{0}+2 k \pi\right) & =\hat{f}\left(m \eta_{0}+2\left(k-j_{0}\right) \pi\right) \\
& =H_{n_{2}-n_{1}}\left(\eta_{0}+2\left(k-j_{0}\right) \pi / m\right) \hat{f}\left(\xi_{0}+2\left(k-j_{0}\right) \pi / m\right) \\
& =H_{n_{2}-n_{1}}\left(\eta_{0}\right) \hat{f}\left(\xi_{0}+2\left(k-j_{0}\right) \pi / m\right) \\
& =\cdots=H_{n_{2}-n_{1}}\left(\eta_{0}\right)^{l} \hat{f}\left(\xi_{0}+2 k^{\prime} \pi\right) \\
& =H_{n_{2}-n_{1}}\left(\eta_{0}\right)^{l} H_{n_{2}-n_{1}}\left(\eta_{0}+2\left(k^{\prime}-j_{0}\right) \pi / m\right) \hat{f}\left(\eta_{0}+2\left(k^{\prime}-j_{0}\right) \pi / m\right) \\
(2.18) & =0 .
\end{aligned}
$$

On the other hand, we see that if $0<j_{0} \leq m-2$, then the union of the sets $U_{l}\left(\eta_{0}\right):=j_{0}+\cdots+m^{l} j_{0}+m^{l} \mathbf{Z} \backslash m^{l+1} \mathbf{Z}, l \geq 0$, is the whole integer set $\mathbf{Z}$, because $\mathbf{Z} \backslash\left(\bigcup_{l=0}^{L} U_{l}\right)=j_{0}+\cdots+m^{L} j_{0}+m^{L+1} \mathbf{Z}$, and $\min \left\{|k|: k \in \mathbf{Z} \backslash\left(\bigcup_{l=1}^{L} U_{l}\right)\right\} \geq$ $\min \left(m^{L+1}-\left(j_{0}+\cdots+m^{l} j_{0}\right),\left(j_{0}+\cdots+m^{L} j_{0}\right)\right) \rightarrow \infty$ as $L \rightarrow \infty$. Therefore $j_{0}=0$ by (2.18) and the linear independent assumption on $f$. This proves that for any $\xi_{0} \in K$ there exists an integer $n$ so that $M^{n} \xi_{0} \in 2 \pi \mathbf{Z}$. Hence $K \subset 2 M^{-N_{1}} \pi \mathbf{Z}$ for some sufficiently large integer $N_{1}$. This together with (2.14) proves that $K_{n} \subset 2 M^{-n} \mathbf{Z}$ for all $n \geq \max \left(N_{0}, N_{1}\right)$, and hence the result follows.

Now we reach the stage of the proof of Theorem 2.1

Proof of Theorem 2.1. Define the correlation of two compactly supported $L^{2}$ functions $f$ and $g$ by

$$
A_{f, g}(\xi):=\sum_{k \in \mathbf{Z}}\langle f, g(\cdot-k)\rangle e^{-i k \xi} .
$$

Recall that $f$ is a refinable function in $L^{2}$, which implies the unit partition property $\sum_{k \in \mathbf{Z}} f(\cdot-k) \equiv 1$. Thus

$$
A_{f, g}(0)=\int_{\mathbf{R}} g(x) d x \neq 0
$$


Denote the set of all $M^{n}$-symmetric roots of $H_{n} A_{f, g}$ by $K_{n}$ with multiplicity being not counted, where as before we say that $a$ and $b$ are two different $M^{n}$ symmetric roots if $a-b \notin 2 M^{-n} \pi \mathbf{Z}$. By (2.20) and Lemma [2.2, $K_{N} \subset 2 M^{-N} \pi \mathbf{Z}$ for some integer $N$. Thus $K_{N}$ is an empty set, since otherwise $0 \in K_{N}$, and then $A_{f, g}(0)=H_{N}(0) A_{f, g}(0)=0$, which contradicts (2.20). From the definition of $K_{N}$ and the assertion $K_{N}=\emptyset$, it follows that $H_{N}(\xi) A_{f, g}(\xi)$ does not have any $M^{N_{-}}$ symmetric root. By the Bézout identity, there exists a trigonometric polynomial $B(\xi)$ so that

$$
\sum_{k=0}^{M^{N}-1} H_{N}\left(\frac{\xi+2 k \pi}{M^{N}}\right) A_{f, g}\left(\frac{\xi+2 k \pi}{M^{N}}\right) \overline{B\left(\frac{\xi+2 k \pi}{M^{N}}\right)}=1 .
$$

Define $h$ by $\hat{h}(\xi)=B\left(\xi / M^{N}\right) \hat{g}\left(\xi / M^{N}\right)$. Obviously $h \in S_{N}(g)$. By (2.6) and (2.21), we have

$$
\begin{aligned}
A_{f, h}(\xi) & =\sum_{k \in \mathbf{Z}} \hat{f}(\xi+2 k \pi) \overline{\hat{h}(\xi+2 k \pi)} \\
& =\sum_{k \in \mathbf{Z}} H_{N}\left(\frac{\xi+2 k \pi}{M^{N}}\right) \overline{B\left(\frac{\xi+2 k \pi}{M^{N}}\right)} \hat{f}\left(\frac{\xi+2 k \pi}{M^{N}}\right) \overline{\hat{g}\left(\frac{\xi+2 k \pi}{M^{N}}\right)} \\
& =\sum_{k=0}^{M^{N}-1} H_{N}\left(\frac{\xi+2 k \pi}{M^{N}}\right) A_{f, g}\left(\frac{\xi+2 k \pi}{M^{N}}\right) \overline{B\left(\frac{\xi+2 k \pi}{M^{N}}\right)} \\
& =1,
\end{aligned}
$$

and hence $h$ is a local dual of $f$.

\section{Poly-SCAle REFinABility}

In this section, we give the proofs of Theorems 1.2 and 1.3. To prove Theorems 1.2 and 1.3 we need a lemma about trigonometric polynomials.

Lemma 3.1. For any given nonzero trigonometric polynomial $d(\xi)$, there exist an integer $N$ and trigonometric polynomials $c_{l}(\xi), 1 \leq l \leq N$, so that

$$
d\left(M^{N} \xi\right)=c_{1}\left(M^{N-1} \xi\right) d\left(M^{N-1} \xi\right)+c_{2}\left(M^{N-2} \xi\right) d\left(M^{N-2} \xi\right)+\cdots+c_{N}(\xi) d(\xi) .
$$

Furthermore, if there does not exist $\xi_{0} \in \mathbf{C}$ so that $d\left(M^{n} \xi_{0}\right)=0$ for all $n \geq 0$, then there exist an integer $N$ and trigonometric polynomials $c_{l}^{*}(\xi), 1 \leq l \leq N$, so that

$$
1=c_{1}^{*}\left(M^{N-1} \xi\right) d\left(M^{N-1} \xi\right)+c_{2}^{*}\left(M^{N-2} \xi\right) d\left(M^{N-2} \xi\right)+\cdots+c_{N}^{*}(\xi) d(\xi) .
$$

Proof. We let $d_{0}(\xi)=d(\xi)$, and inductively for $n \geq 1$, we let $d_{n}(\xi)$ be the trigonometric polynomial with minimal degree so that any common factor between $d(\xi)$ and $d_{n-1}(\xi)$ is a factor of $d_{n}(M \xi)$. Here the degree of a trigonometric polynomial $p(\xi):=\sum_{k=k_{1}}^{k_{2}} p(k) e^{-i k \xi}$ with $p\left(k_{2}\right) p\left(k_{1}\right) \neq 0$ is defined by $\operatorname{deg} p=k_{2}-k_{1}$. Note that $d_{n}(\xi)$ is a factor of $d_{n-1}(\xi / M) \cdots d_{n-1}(\xi / M+2(M-1) \pi / M)$ since $d_{n-1}(\xi)$ is a factor of the trigonometric polynomial $d_{n-1}(\xi) \cdots d_{n-1}(\xi+2(M-1) \pi / M)$. Then the degree of $d_{n}$ is less than that of $d_{n-1}$, which implies that there exists an integer $N$ so that the degree of $d_{n}$ is the same as the degree of $d_{N}$ for all $n \geq N$. Recall that $d_{n+1}(M \xi)$ is a factor of $d_{n}(\xi) \cdots d_{n}(\xi+2(M-1) \pi / M)$. Then comparing the degrees of the above two trigonometric polynomials, we get

$$
d_{n+1}(M \xi)=c_{n}(\xi) d_{n}(\xi) \cdots d_{n}(\xi+2(M-1) \pi / M)
$$


where $c_{n}(\xi), n \geq N$, are nonzero monomials. This together with the construction of $d_{n+1}$ implies that

$$
d_{n}(\xi) \text { is a factor of } d(\xi) \text { for all } n \geq N .
$$

Therefore there exists a trigonometric polynomial $c_{0}$ so that

$$
d(\xi)=c_{0}(\xi) d_{N}(\xi)
$$

From the construction of $d_{n}$, there exist trigonometric polynomials $a_{n}$ and $b_{n}$ so that

$$
d_{n}(M \xi)=a_{n}(\xi) d(\xi)+b_{n}(\xi) d_{n-1}(\xi)
$$

for all $1 \leq n \leq N$. The assertion (3.1) follows from (3.5) and (3.6).

Now we prove (3.2). Let $d_{n}, n \geq 0$, and $N$ be as above. By (3.6), it suffices to show that $d_{N}$ is a monomial when there does not exist $\xi_{0} \in \mathbf{C}$ so that $M^{n} \xi_{0}, n \geq 0$, are roots of $d(\xi)$. Suppose, on the contrary, that $d_{N}$ is not a monomial. Then $d_{N}\left(\eta_{1}\right)=0$ for some $\eta_{1} \in \mathbf{C}$. Iteratively using (3.3), we obtain $d_{n+N}\left(M^{n} \eta_{1}\right)=0$ for all $n \geq 0$. This together with (3.4) implies that $d\left(M^{n} \eta_{1}\right)=0$ for all $n \geq 0$, which is a contradiction.

Now we start to prove Theorems 1.2 and 1.3 .

Proof of Theorem 1.2. Write $g=\sum_{k \in \mathbf{Z}} d(k) f(\cdot-k)$ for some $\{d(k)\} \in \ell_{0}$, and assume that $f$ satisfies the refinement equation $f=\sum_{k \in \mathbf{Z}} a(k) f(M \cdot-k)$ for some $\{a(k)\} \in \ell_{0}$. Then setting $H(\xi)=\frac{1}{M} \sum_{k \in \mathbf{Z}} a(k) e^{-i k \xi}$ and $d(\xi)=\sum_{k \in \mathbf{Z}} d(k) e^{-i k \xi}$ yields

$$
\hat{f}(M \xi)=H(\xi) \hat{f}(\xi) \quad \text { and } \quad \hat{g}(\xi)=d(\xi) \hat{f}(\xi) .
$$

By Lemma 3.1, there exist a positive integer $N$ and trigonometric polynomials $c_{n}(\xi), 1 \leq n \leq N$, so that

$$
d\left(M^{N} \xi\right)=\sum_{n=1}^{N} c_{n}\left(M^{N-n} \xi\right) d\left(M^{N-n} \xi\right)
$$

Multiplying $\hat{f}\left(M^{N} \xi\right)$ at both sides of (3.8) and using (3.7), we obtain

$$
\begin{aligned}
\hat{g}\left(M^{N} \xi\right) & =\sum_{n=1}^{N} c_{n}\left(M^{N-n} \xi\right) d\left(M^{N-n} \xi\right) \hat{f}\left(M^{N} \xi\right) \\
& =\sum_{n=1}^{N} c_{n}\left(M^{N-n} \xi\right) H\left(M^{N-1} \xi\right) \cdots H\left(M^{N-n} \xi\right) \hat{g}\left(M^{N-n} \xi\right) .
\end{aligned}
$$

Hence taking the inverse Fourier transform at both sides of (3.9) proves that $g$ is poly-scale refinable.

Proof of Theorem 1.3. We use the same argument as in the proof of Theorem 1.2 except for the equation (3.8) being replaced by (3.2). We omit the details of the proof here. 


\section{Generalization, applications and problems}

In this section, we give a few remarks about generalization, applications and open problems related to Theorems 1.11 .2 and 1.3 .

For a vector-valued compactly supported function $F=\left(f_{1}, \ldots, f_{r}\right)^{T}$ on $\mathbf{R}^{d}$, we define the semi-convolution $F *^{\prime}$ by

$$
F *^{\prime}:(\ell)^{r} \ni\{c(k)\} \longmapsto \sum_{k \in \mathbf{Z}^{d}} c(k)^{T} F(\cdot-k),
$$

where $(\ell)^{r}$ denotes the $r$ copies of the space of all sequences on $\mathbf{Z}^{d}$, and the shiftinvariant spaces $S_{n}(F)$ by

$$
S_{n}(F)=\left\{F *^{\prime} c\left(M^{n} \cdot\right): c \in\left(\ell_{0}\right)^{r}\right\} .
$$

We say that $F$ is $M$-refinable if

$$
S_{0}(F) \subset S_{1}(F)
$$

and poly-scale refinable if

$$
S_{0}(F) \subset S_{1}(F)+\cdots+S_{N}(F)
$$

for some $N \geq 1$. For two vector-valued compactly supported functions $F=$ $\left(f_{1}, \ldots, f_{r}\right)^{T} \in L^{2}$ and $G=\left(g_{1}, \ldots, g_{r}\right)^{T} \in L^{2}$, we say that $G$ is a local dual of $F$ if $\left\langle f_{i}(\cdot-k), g_{i^{\prime}}\left(\cdot-k^{\prime}\right)\right\rangle=\delta_{i i^{\prime}} \delta_{k k^{\prime}}$ for all $1 \leq i, i^{\prime} \leq r$ and $k, k^{\prime} \in \mathbf{Z}^{d}$.

First we consider the generalization of Theorem 1.1 to the vector-valued case.

Theorem 4.1. Let $F=\left(f_{1}, \ldots, f_{r}\right)^{T}$ and $G=\left(g_{1}, \ldots, g_{r}\right)^{T}$ be vector-valued compactly supported functions in $L^{2}$. Assume that $F$ is $M$-refinable and has linear independent shifts, the symbol $H(\xi)$ of the refinable function $F$ satisfies $\operatorname{det} H(\xi) \not \equiv 0, G$ satisfies $\int_{\mathbf{R}} G(x) d x \neq 0$, and the correlation matrix $\sum_{k \in \mathbf{Z}} \widehat{F}(\xi+2 k \pi) \overline{\widehat{G}(\xi+2 k \pi)^{T}}$ is nonsingular for some $\xi_{0} \in \mathbf{C}$. Then there exist functions $h_{1}, \ldots, h_{r} \in S_{N}(g)$ for some $N \geq 0$ so that $\left(h_{1}, \ldots, h_{r}\right)^{T}$ is a local dual of $F$.

As an easy consequence of Theorem 4.1 we see that for a vector-valued refinable function $F \in L^{2}$ having linear independent shifts, if the corresponding symbol has nonzero determinant, then a local dual to $F$ can be found in $S_{N}(F)$ for some $N \geq 1$. We do not include the proof of Theorem 4.1 here since the proof is similar to the one of Theorem 2.1, except for more delicate analysis about symmetric roots of a matrix-valued trigonometric polynomial.

Next we consider the compactly supported distributional solution of a poly-scale refinement equation. As mentioned in [7], for a poly-scale refinement equation, there is a corresponding vector-valued refinement equation. In particular, let $F=$ $\left(f_{1}, \ldots, f_{r}\right)^{T}$ satisfy the following poly-scale refinement equation,

$$
F=\sum_{n=1}^{N} \sum_{k \in \mathbf{Z}} c_{n}(k) F\left(M^{n} \cdot-k\right)
$$

where $\left\{c_{n}(k)\right\} \in \ell_{0}^{r \times r}, 1 \leq n \leq N$, set

$$
H_{n}(\xi)=M^{-n} \sum_{k \in \mathbf{Z}} c_{n}(k) e^{-i k \xi}, 1 \leq n \leq N,
$$


and define

$$
\tilde{H}(\xi)=\left(\begin{array}{ccccc}
H_{1}\left(M^{N-1} \xi\right) & H_{2}\left(M^{N-2} \xi\right) & \cdots & H_{N-1}(M \xi) & H_{N}(\xi) \\
I & 0 & \cdots & 0 & 0 \\
0 & I & \cdots & 0 & 0 \\
\vdots & \vdots & \ddots & \vdots & \vdots \\
0 & 0 & \cdots & 0 & 0 \\
0 & 0 & \cdots & I & 0
\end{array}\right) .
$$

Then the vector-valued function $\tilde{F}$ defined by

$$
\widehat{\tilde{F}}(\xi)=\left(\begin{array}{c}
\widehat{F}\left(M^{N-1} \xi\right) \\
\vdots \\
\widehat{F}(M \xi) \\
\widehat{F}(\xi)
\end{array}\right)
$$

satisfies the refinement equation

$$
\widehat{\tilde{F}}(\xi)=H(\xi / M) \widehat{\tilde{F}}(\xi / M) .
$$

As an application of Theorem 2.1 in [11, we have the following result about the existence of a nonzero compactly supported distributional solution of a poly-scale refinement equation.

Theorem 4.2. Let $\left\{c_{l}(k)\right\} \in \ell_{0}^{r \times r}, 1 \leq l \leq N$, and set

$$
H(z, w)=\sum_{l=1}^{N} \sum_{k \in \mathbf{Z}} c_{l}(k) z^{k} w^{l} .
$$

Then there exists a nonzero compactly supported distributional solution $F$ of the poly-scale refinement equation

$$
F=\sum_{n=1}^{N} \sum_{k \in \mathbf{Z}} c_{n}(k) F\left(M^{n} \cdot-k\right)
$$

if and only if $H\left(1, M^{-l}\right)-I$ is singular for some nonnegative integer $l$.

Now we consider the possible application of Theorem 1.3. Let $f$ be a compactly supported refinable function on the real line, and let $g \in S_{0}(f)$. By Theorem 1.3 there exist a positive integer $N$ and sequences $\left\{c_{n}(k)\right\} \in \ell_{0}, 1 \leq n \leq N$, so that $f=\sum_{n=1}^{N} \sum_{k \in \mathbf{Z}} c_{n}(k) g\left(M^{n} \cdot-k\right)$. Then taking the inner product with an $L^{2}$ function $h$ leads to

$$
\langle h, f(\cdot-k)\rangle=\sum_{n=1}^{N} \sum_{l \in \mathbf{Z}} c_{n}(l)\left\langle h, g\left(M^{n} \cdot-M^{n} k-l\right)\right\rangle \quad \text { for all } k \in \mathbf{Z},
$$

which implies that the local information of the sequence $\{\langle h, f(\cdot-k)\rangle\}$ can be obtained from the local information of $\left\{\left\langle h, g\left(M^{n} \cdot-k^{\prime}\right)\right\rangle\right\}$ at the finer level $n$ between 1 and $N$. The above formula is useful in the reconstruction of the original function or signal in the shift-invariant space generated by $f$, especially when it is more costly for designing a good average sampler $g$ to obtain the data at an initial level than for using an easy average sampler $g$ to obtain the data at a finer level. 
Finally, we propose some problems about local dual and poly-scale refinability:

Problem 1. Let $F$ and $G \in L^{2}$ be vector-valued compactly supported refinable functions on $\mathbf{R}^{d}, d \geq 2$, and have linear independent shifts. Can we find a local dual of $F$ in $S_{N}(G)$ for some $N \geq 1$.

Problem 2. Let $F=\left(f_{1}, \ldots, f_{r}\right)^{T}$ be a vector-valued compactly supported (polyscale) refinable function having linear independent shifts. Characterize all functions $g_{1}, \ldots, g_{r} \in S_{0}(F)$ that are poly-scale refinable.

Problem 3. Let $F=\left(f_{1}, \ldots, f_{r}\right)^{T}$ be a vector-valued compactly supported refinable function having linear independent shifts. Characterize all functions $g_{1}, \ldots, g_{r}$ $\in S_{0}(F)$ so that

$$
S_{0}(F) \subset S_{1}(G)+\cdots+S_{N}(G)
$$

for some $N \geq 1$, where $G=\left(g_{1}, \ldots, g_{r}\right)^{T}$.

\section{REFERENCES}

[1] A. Ben-Artzi and A. Ron, On the integer translates of a compactly supported function: dual bases and linear projectors, SIAM J. Math. Anal., 21(1990), 1550-1562. MR1075591 (91j:41009)

[2] C. K. Chui, "An Introduction to Wavelets", Academic Press, 1992. MR1161244 (92k:42001)

[3] C. K. Chui, W. He and J. Stöckler, Compactly supported tight and sibling frames with maximum vanishing moments, Appl. Comput. Harmon. Anal., 13(2002), 224-262. MR 1942743 (2004a:94011)

[4] C. K. Chui, W. He, J. Stöckler and Q. Sun, Compactly supported tight affine frames with integer dilations and maximum vanishing moments, Adv. Comput. Math., 18(2003), 159-187. MR 1968118 (2004d:42050)

[5] I. Daubechies, "Ten Lectures on Wavelets", CBMS-NSF Regional Conference Series in Applied Mathematics, 61, SIAM, Philadelphia, 1992. MR1162107 (93e:42045)

[6] I. Daubechies, B. Han, A. Ron and Z. Shen, Framelets: MRA-based constructions of wavelet frames, Appl. Comput. Harmon. Anal., 14(2003), 1-46. MR1971300 (2004a:42046)

[7] S. Dekel and N. Dyn, Poly-scale refinability and subdivision, Appl. Comput. Harmon. Anal., 13(2002), 35-62. MR1930175 (2003h:42057)

[8] G. C. Donovan, J. S. Geronimo, and D. P. Hardin, Intertwining multiresolution analyses and the construction of piecewise-polynomial wavelets, SIAM J. Math. Anal., 27(1996), 17911815. MR 1416519 (98c:42029)

[9] P. G. Lemarié-Rieusset, On the existence of compactly supported dual wavelets, Appl. Comput. Harmon. Anal., 4(1997), 117-118. MR1429683 (97h:42018)

[10] R.-Q. Jia and C. A. Micchelli, On linear independence for integer translates of a finite number of functions, Proc. Edinburgh Math. Soc., 36(1993), 69-85. MR1200188 (94e:41044)

[11] Q. Sun, Compactly supported distributional solutions of nonstationary nonhomogeneous refinement equations, Acta Math. Sinica, 17(2001), 1-14. MR.1831741 (2002d:42037)

[12] Q. Sun, N. Bi and D. Huang, "An Introduction to Multiband Wavelets", Zhejiang University Press, China, 2001.

[13] A. Ron, A necessary and sufficient condition for the linear independence of the integer translates of a compactly supported distribution, Constr. Approx., 5(1989), 297-308. MR0996932 (90g:41019)

[14] K. Zhao, Global linear independence and finitely supported dual basis, SIAM J. Math. Anal., 23(1992), 1352-1355. MR1177795 (93h:41034)

Department of Mathematics, University of Central Florida, Orlando, Florida 32816

E-mail address: qsun@mail.ucf.edu 